\title{
Design of Wide Voltage and High Efficiency Equalization Charging Control System
}

\author{
Suping Li, Zaiwu Zhu* and Huijiong Li \\ School of Mechanical and Electronic Engineering, Chaohu College, Chaohu 238000,China. \\ lsp20061002@126.com
}

\begin{abstract}
A dissipative equalization circuit system is designed in order to ensure battery performance and prolong battery life.The equalization circuit system has wide voltage and high efficiency performance. It achieved intelligent equalizing charging by comparing battery terminal voltage and the setting threshold voltage and taking use of constant voltage load module and constant current source module. When the battery terminal voltage is less than the threshold voltage, the battery enters the constant current charging state. When the battery terminal voltage is greater than the threshold voltage, the battery enters the constant load model.When MCU detects that all currents flow through the constant load module, the charging process is determined.The circuit structure is simple and efficient.
\end{abstract}

Keywords: Wide Voltage, High Efficiency, Equalization Circuit

\section{INTRODUCTION}

At present, the main factors that affect the promotion and application of electric vehicles include the safety of the power battery and the cost. Extending battery life is one of the effective ways to reduce the cost. To ensure battery performance and prolong battery life, it is necessary to manage and control the battery reasonably and effectively. Intelligent charging and discharging technology is the key. A large number of human and material resources have been devoted to carry out the extensive and in-depth research at home and abroad ${ }^{[1,2]}$. Since 1997, foreign research institutes have been conducting research on the practical application of battery management system which have made a lot of achievements ${ }^{[3,4]}$. At present, there is a rich variety of equalization circuit topology, but in general, it can be divided into two categories: energy consumption equalization circuit and energy non-consumption equalization circuit.The energy consumable equalization circuit structure is simple, the control strategy is not complex, and the discharge speed is fast, which can discharge multiple battery cells simultaneously ${ }^{[5,6]}$. The energy non-consumable equalization circuit usually requires a certain equilibrium algorithm, which is different for different battery. The control algorithm is complex.

Considering the above technical status, a kind of energy consumable equalization system based on constant pressure load module is designed. The battery is balanced with the single battery voltage and charging voltage threshold. The equalization circuit is simple and efficiency.

\section{GENERAL FRAMEWORK}

In this paper a method of decentralized data acquisition and centralized data processing is proposed. The voltage, current and temperature acquisition circuit is designed and the algorithm is accomplished through MCU module of the central control unit. The structure diagram is shown in figure 1.

The single chip microprocessor real-time processing the data collected by the battery data collector. When the battery voltage is detected below the set voltage threshold, the relay starts the constant current charging circuit and the system enter charging model. When the voltage of a battery is not less than the set voltage threshold, the MCU module will control the relay for constant-voltage discharging battery. The relay is connected to the constant pressure load in parallel with the discharging battery and the current no longer flows through the fully charged battery. When the single-chip microcomputer detects that all current is through the constant pressure load module, the balance charge is finished.The system has the characteristics of low cost, high discharge efficiency and high 
reliability.

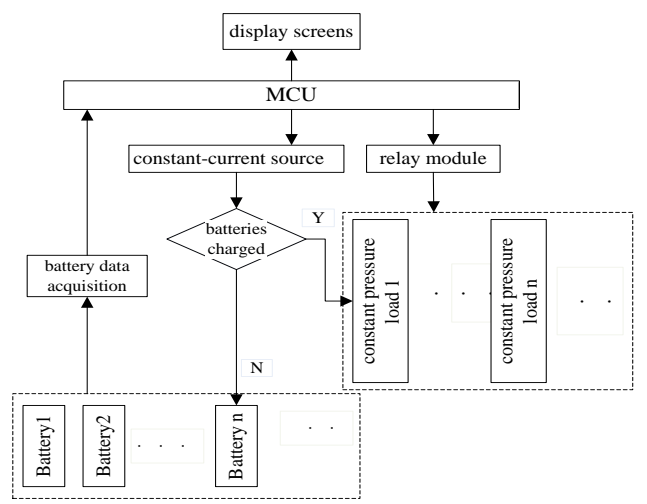

FIGURE 1. System structure diagram.

\section{KEY TECHNOLOGY}

The key technologies of the equalization charging-discharging system are charging and discharging equalization technology, wide voltage high-efficiency charging technology and the temperature management circuit.

\subsection{Charging and Discharging Equalization Technology}

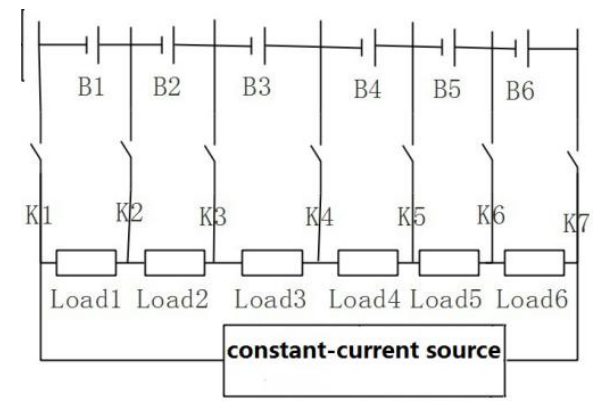

FIGURE 2. Schematic diagram of equalization charging circuit.

In the charging and discharging equalization circuit, the constant current source module is charging circuit module, B means battery, and Load means the constant voltage load module. Each constant pressure load is set with a balanced voltage threshold. K means relay. After the system is charged, firstly detect whether each battery voltage is within the set range. When the battery voltage is below the threshold voltage, the relay is connected to charge the corresponding battery. The constant voltage load module begins to work when the battery voltage exceeds the voltage threshold. When MCU detects that current flows through constant voltage load module only, the equalization charge and discharge of the battery pack is completed.This process realizes the equalization chargingdischarging of the battery pack.

\subsection{Wide Voltage High Efficiency Charging Circuit}

The design of wide voltage high-efficiency charging technology firstly considering the connection between charging current and battery power while the battery power is varying.Secondly, there are fluctuations in power grid voltage. Both of above factors will affect the current output value of the charging circuit and then the stability of battery charging state is affected. Therefore, the transformer circuit needs wide voltage output to ensure that the constant current source module can output the dynamic current value.

In specific design,the transformer circuit is composed of multiple relays to make a tap selection circuit.This relay connects different voltage taps to achieve wide voltage output. Figure 2 shows the voltage of each tap .The three relays constitute a tap selection circuit. The system can choose $32 \mathrm{~V}, 36 \mathrm{~V}, 40 \mathrm{~V}$ and $44 \mathrm{~V}$. Switching different input 
voltages according to different needs to maintain proper voltage drop on the regulating power tube. It can not only meet the demand of constant current charging but also reduce the heating power consumption and achieve higher charging efficiency.

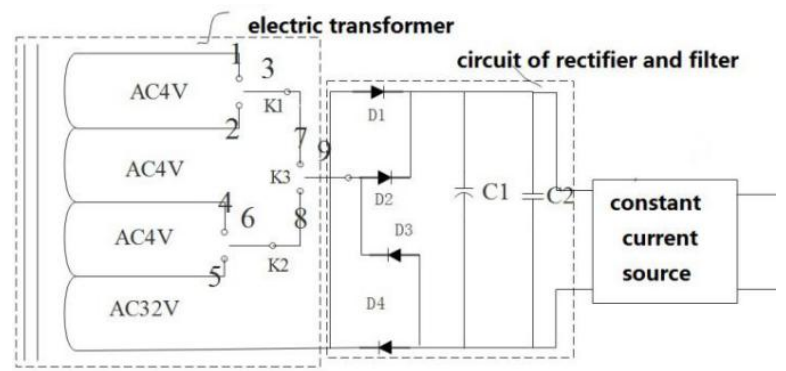

FIGURE 3. Wide voltage and high-efficient charging circuit diagram.

\subsection{Temperature Management Module}

This paper has a soft and hard integrated temperature control system. The temperature sensor is configured in the hardware circuit, and the constant voltage load circuit module and the single battery temperature are collected to protect system temperature. The power tube of the constant pressure load module uses TO247 to enhance the cooling effect and long working safety and stability.

\section{SYSTEM WORKFLOW}

The system hardware design must realize the rational management of the power battery pack. Firstly, the accuracy of the collected data must be guaranteed.Secondly is reliability and stability of system communication.Last but not least is the anti-interference feature. The system integral design needs integrating hardware-design and software-design.The data type of battery pack is determined depending on the hardware design.The design of hardware circuit parameters is determined according to the acquisition parameters and precision requirements.A reasonable communication interface circuit is designed according to the anti-interference design. The charging process of a battery is shown in figure 4 .

The same charging operation is carried out for each battery in battery pack during the charge and discharge process. Firstly, the battery polarity and terminal voltage are checked after the system initialized. When the battery polarity is not reversed and the battery terminal voltage is less than the set voltage threshold, the single battery begins to charge. For the battery pack balance charging and discharging process, when the last battery is not full, do not charge the next battery.Checking to see if the individual battery is full until all the batteries are full, and the battery pack charging ends.

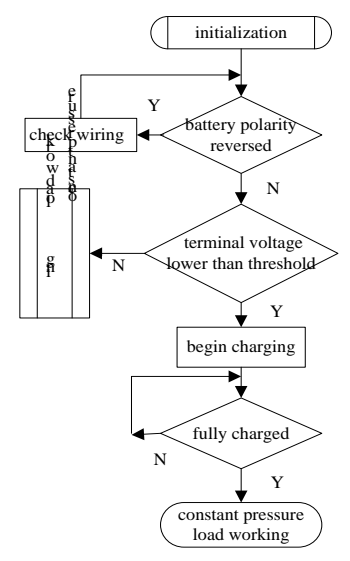

FIGURE 4. Charging process of the battery. 


\section{EXPERIMENTAL RESULTS AND ANALYSIS}

After the welding of the equalizing charging circuit is completed, the charging process of the battery pack is tested, and the test results are shown in table 1.

TABLE 1. Charging circuit parameters.

\begin{tabular}{ccc}
\hline Numbler & Scheme 1 & Scheme $\mathbf{2}$ \\
\hline 1 & single battery nominal voltage & $3.7 \mathrm{~V}$ \\
2 & single battery balanced voltage & $3.48 \mathrm{~V}$ \\
3 & maximum balance discharge current & $6 \mathrm{~A}$ \\
4 & balance charge current & $0-5 \mathrm{~A}$ tunable, error $\pm 0.2 \mathrm{~A}$ \\
5 & working power & AC198 $\sim 242 \mathrm{~V} / 50 \mathrm{~Hz}$ \\
6 & the number o monitoring battery packs & $1 \sim 12$ set \\
7 & the number of monitoring battery & $1 \sim 10$ \\
\hline
\end{tabular}

The charging module of the charging circuit adopts the wide voltage design so the constant charging current can be provided when the working power supply fluctuates in a certain range. The system can withstand the work power of AC $198-242 \mathrm{v} / 50 \mathrm{~Hz}$. Considering that the charging current needs to be changed in practical application, the charging current in this design can be adjusted within 0-5A. In addition,the design also has the following characteristic:The two-stage intelligent charging technology is adopted, the initial constant current charging and the late constant voltage discharging. The system also has multiple alarm functions.

\section{CONCLUSIONS}

The equalization charging system sets up data collector to detect the voltage of each battery in real time, ensuring that each circuit is pressed in the appropriate range. This is a software protection for battery pack. Constant current charging is adopted in the system, and the voltage overcharge of constant pressure load belongs to the category of hardware protection. The system adopts the dual protection measures of hardware protection combined with software protection which ensures the safe and modular realization of the battery and charging equipment. The system has the characteristics of low cost and high efficiency.

\section{ACKNOWLEDGMENTS}

This work was financially supported by Wanwei science and technology innovation incubation fund project (WWZR-201715) and national university student innovation and entrepreneurship project (201710380021) .

\section{REFERENCES}

1. Oman H, Electric car progress, Aerospace and electronic systems magazine. 17(6) (2002)30-35.

2. Arai, J, et al. Development of a high power lithium secondary battery for hybrid electric vehicles, Journal of power sources. 146(1-2) (2005)788-792.

3. Gaizhen Y,Chaosheng X, etal, Overview of battery equalization technology for electric vehicles, Journal of chongqing institute of science and technology (natural science edition).16(1)(2014)130-133.

4. Suoyu L. Research on balance technology of power lithium battery pack, beijing giaotong university.2011.

5. Chunyan,X, Chengjun, $X$ and Feng,J. Research and design of the balanced technology of battery management system in wind power generation. Int.ControlAutom.2016,453-462.

6. Tschöke, H.: Die Elektrifizierung des Antriebsstrangs, Berlin: Springer, 2015 Gellerich, W.: Akkumulatoren — Grundlagen und Praxis. Herzogenrath: Shaker Media, 2015. 